\title{
Process preparation resources integration service mode and semantic description in manufacturing grid
}

\author{
Tong Yifei ${ }^{1, ~ a ~, ~ L i ~ D o n g b o ~}{ }^{2, b}$ and He Yong ${ }^{3, c}$ \\ ${ }^{1}$ Nanjing University of Science and Technology, School of Mechanical Engineering 402, 210094 \\ Nanjing, People's Republic of China \\ ${ }^{2}$ Nanjing University of Science and Technology, School of Mechanical Engineering 402, 210094 \\ Nanjing, People's Republic of China \\ ${ }^{3}$ Nanjing University of Science and Technology, School of Mechanical Engineering 402, 210094 \\ Nanjing, People's Republic of China
}

aemail: tyf51129@yahoo.com.cn(corresponding author), bemail: db_callar@yahoo.com.cn, cemail: yhe1964@mail.njust.edu.cn

Keywords: manufacturing grid; process preparation; resources modeling; XML.

\begin{abstract}
Manufacturing enterprises are now facing serious challenges and pressures from the tide of globalization. After analyzing the features of process preparation resource, the integration service mode and semantic description for process preparation based on manufacturing grid is studied. Firstly, process preparation resources are defined and classified. Secondly, information modeling of process preparation in manufacturing grid is discussed. Thirdly, the integrated service mode of process preparation resources in manufacturing grid is discussed. Finally, an example of one kind of process preparation resource is given to illustrate the resource semantic description based on XML. Above research is benefit for application of manufacturing grid and the information progress of national defense industry.
\end{abstract}

\section{Introduction}

In 2000, grid was defined as "resource sharing and coordinated problem solving in dynamic, multi-institutional virtual organizations" by Ian Foster [1]. Most grid researches focused on scientific computing while little on engineering application esp. manufacturing field. Due to the abundance, high integration, coordinated utilization and virtualization of manufacturing resources, the application of grid technologies, which focus on dynamic, cross-organizational sharing and collaborative work [1], in manufacturing field is promising.

Process preparation is an important interface between computer aided design (CAD) and computer aided manufacturing (CAM) in the computer integrated manufacturing environment. Process preparation activity involves widely including product structure manufacturability evaluation, technological process design, tooling design, production process preparation and production process organization etc. [2], the core of which is to manage and take full advantage of enterprise information resources so as to raise the rapid response ability and economic utility of enterprises.

In terms of resource modeling, many models have been presented aiming at variant data exchange standard, aided modeling technique, model type, modeling environment and so on. All the above researches seek to carry out rapid process preparation by intellectual reasoning, resources sharing and collaborative work. However, with the complexity of enterprise process and resources increasing the speed of computing will be the bottleneck problem. While grid can operate computing systems thousands of times as fast as conventional computers and promote resources utilization by sharing resources of wide area and decreasing redundancy[3].

The intention of this present paper is to study the resource modeling for process preparation based on manufacturing grid. In the next section, process preparation resources are defined and classified. Modeling of process preparation in manufacturing grid is discussed in 3rd section. The application flow and xml-based semantic description of process preparation resources are discussed separately in section 4 th and 5 th section. Finally the future work will be pointed out. 


\section{Definition and classification of process preparation resource}

"Process preparation" is the link between CAD system and CAM system and its resources cover a wide range and variety. In our research, process preparation resources in manufacturing grid are defined as combined factors of economic activity in product life cycle involved in the process of product design, manufacturing and production organization, including process preparation hardware and software during product life cycle and information and knowledge supporting resource sharing and collaborative process preparation. Based on years of researches and applications of the work in the manufacturing enterprises, the process preparation resources in manufacturing grid are divided into 5 types as shown in Fig.1.

$>$ Software resources can be classified into of design software and management software according to practical application. Design software mainly include computer-aided design (CAD, such as Pro / E, SolidWorks, AutoCAD, UG, etc.), reverse engineering software, computer aided process planning (CAPP), computer-aided manufacturing (CAM) and computer-aided engineering (CAE, such as ADAMS, NASTRAN, PATRAN, etc.). Management software is used to provide necessary supports for resource management and use, mainly including product data management (PDM), enterprise resource management (ERP), supply chain management (SCM) and so on.

$>$ Equipment resources mainly refer to machining center / machine tools, rapid prototyping equipment, machine workstations and so on.

$>$ Document resources refer to the process files during the collaborative process preparation and the intermediate operating results and knowledge stored in or as files.

$>$ Processor and storage system resources are those resources with specific performance indicators which can meet the needs of high computing efficiency, high storage capacity and so on.

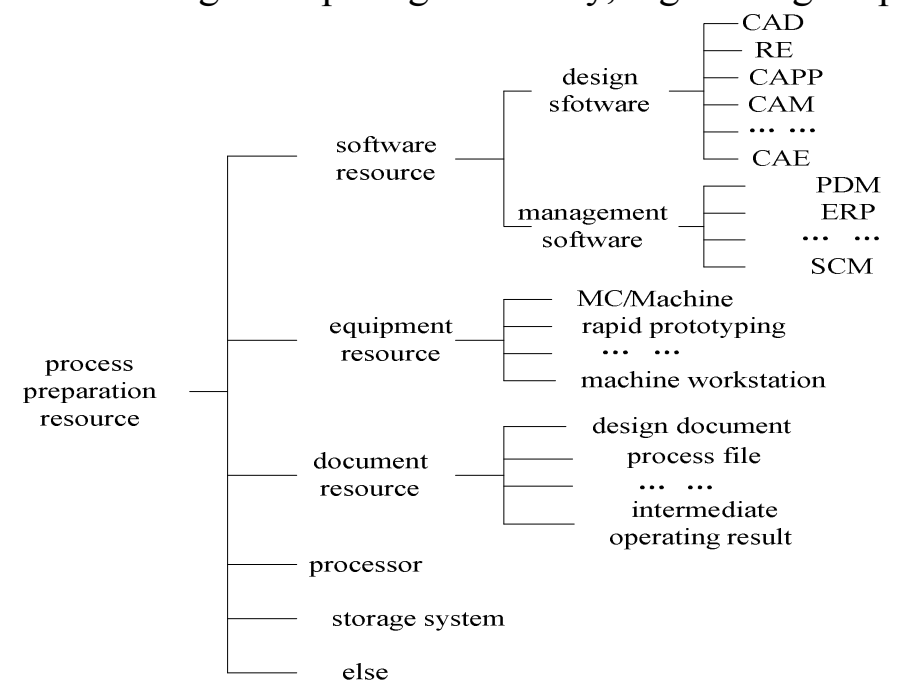

Fig. 1 Process preparation resource classification

\section{Modeling of process preparation resource}

Process preparation resources in grid environment can be characterized by: (1) rich resources; (2) high integrated resources; (3) optimized collaboration; (4) resources virtualization; (5) dynamic use of resources; (6) transparent service etc. [4, 5]. Owing to these characteristics, grid users must be fully aware of grid resource information before using grid resources. Therefore, resource information should be abstracted and represented in a uniform style. The attributes of a grid resource consists of two parts: (1)static attributes, which include resource management attributes (such as name, type, code, etc.), functional attributes, performance attributes, usage attribute (such as input-output type), etc.; (2) dynamic attributes, which can reflect the resource changes over time, including status attributes, load attributes, the current computing capabilities and so on. 
Considering the above five kinds of process preparation resources in grid environment: software resources, equipment resources, document resources, processor and storage system, each type of resources can be further subdivided and represented uniformly. The above five categories of resources are modeled as follows:

$>$ Software resources / equipment resources as shown in Fig.2: the static attributes of software resources / equipment resources describe resource management characteristics (such as: resource name, type, remark, code, etc.), functions and sub-functional characteristics (such as: three dimensional modeling, document management, etc.), performance characteristics (such as machine power, interface standards, etc.) and usage characteristics (input / output type, online / offline, etc.). On the other hand, the dynamic attributes of software resources / equipment resources are used to describe the status characteristics (including initialization, used, waiting, hung up, etc.), the current computing capability (Note: Here the "computing " is a broad concept, including computing, processing, storage and other forms), task list and resources evaluation by grid users. "

$>$ Document resources as shown in Fig.3: the static attributes of document resources describe the management features (such as: file name, type, version information, etc.), file path, a brief file description, and so on. On the other hand, the dynamic attributes of document resources are used to describe the current number of connections and the maximum number of connections, files usage list and access control permissions.

$>$ Processor resources as shown in Fig. 4 also describe the static attributes of processor type, frequency, and operating system and the dynamic attributes of current processing capacity, external communication bandwidth, load and load threshold.

$>$ Storage resources as shown in Fig.5 describe the static attributes of resource type, path and operating system; and dynamic attributes of current capacity, load and load threshold.

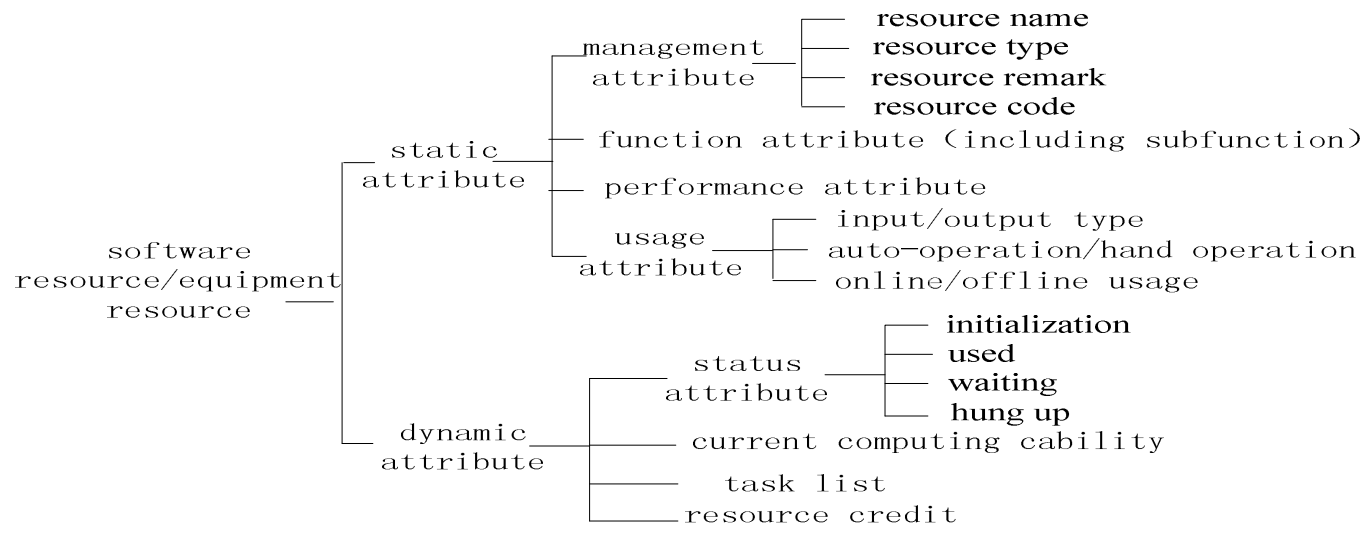

Fig.2 Software resource/equipment resource attributes

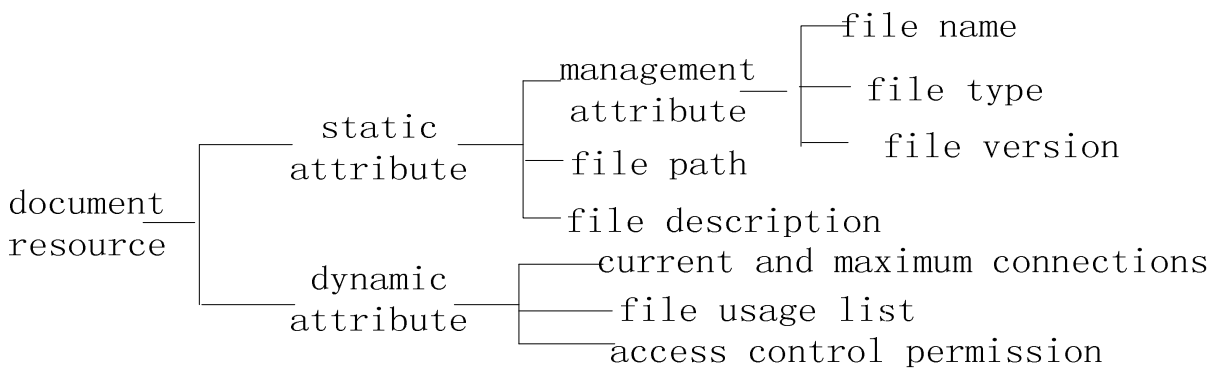

Fig.3 Document resource attributes 


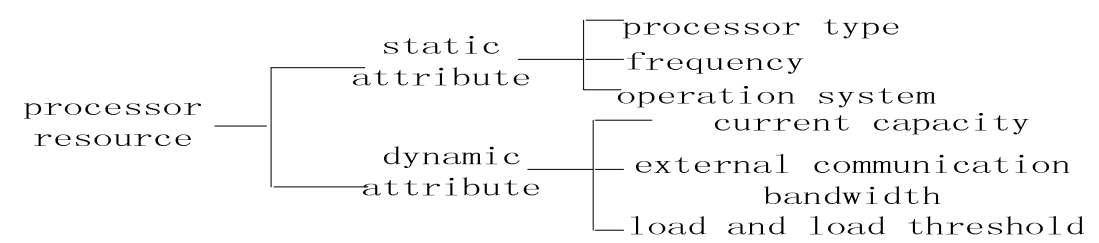

Fig.4 Processor resource attributes

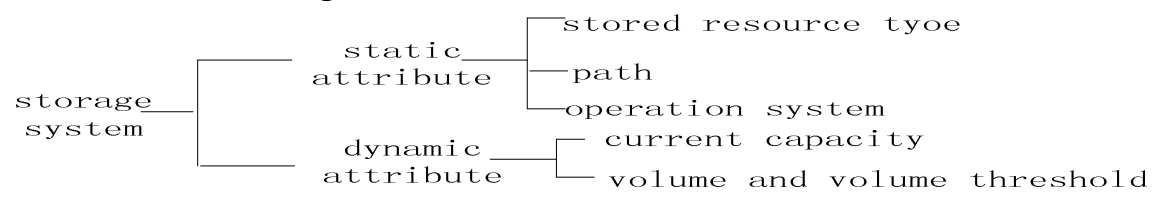

Fig. 5 Storage system attributes

\section{Process preparation resource integrated service mode}

The application procedure of process preparation in manufacturing grid was presented in our former research as show in fig.6. Obviously the mapping between tasks, workflow and service nodes is the basis of application. Users often seek specific process data, which can be obtained by configuring available application components and executing them on the Grid [6]. So user requirements and resources description are the preconditions of application, which can determine corresponding solution by different components due to their specialization and diversity.

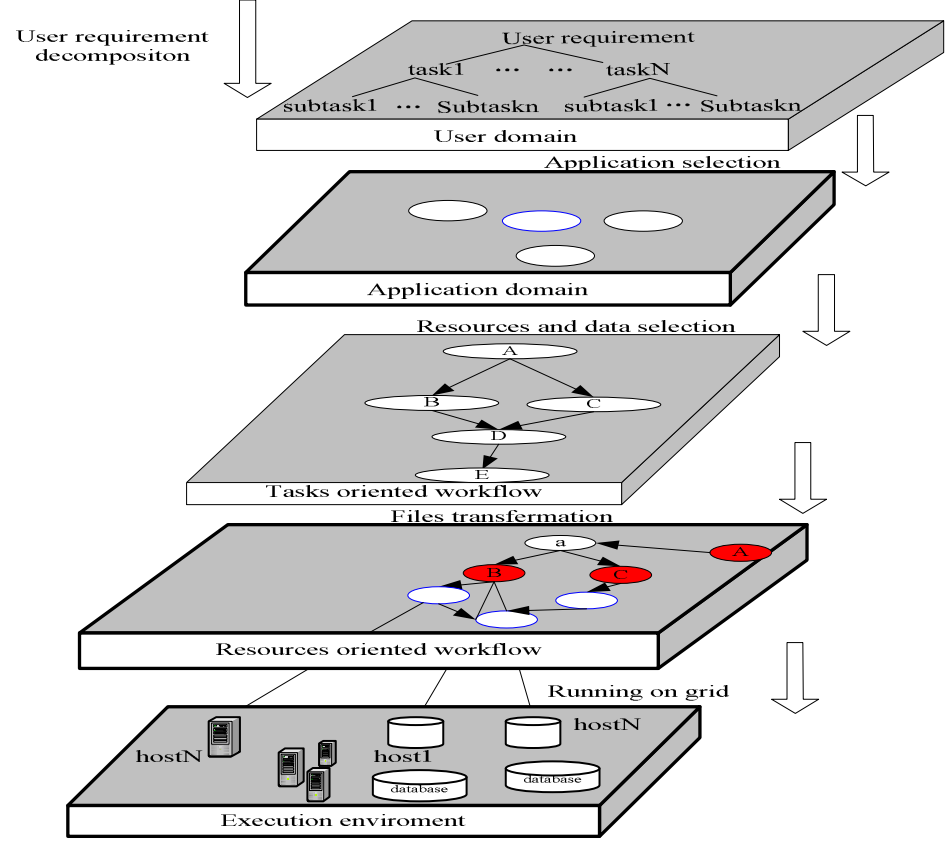

Fig.6 Application procedure of process preparation in manufacturing grid

\section{XML-based process preparation resources information model semantic description}

In our research, the above process preparation resources information model is described by defining a detailed XML Schema description of resource properties. The following shows a simplified XML description example of one kind of grid resources:

$<$ VirtualOrganization1Host $>$

$<$ VirtualOrganization1 $>$

$<$ Resource $>$

$<$ Software $>$

$<$ SoftwareA $>$

$<$ SoftwareName $>$ Solidworks $<$ /SoftwareName $>$

$<$ SoftwareType $>$ DESIGN $</$ SoftwareName $>$

$<$ Function $>$ "3D modeling" $<$ /Function $>$ 


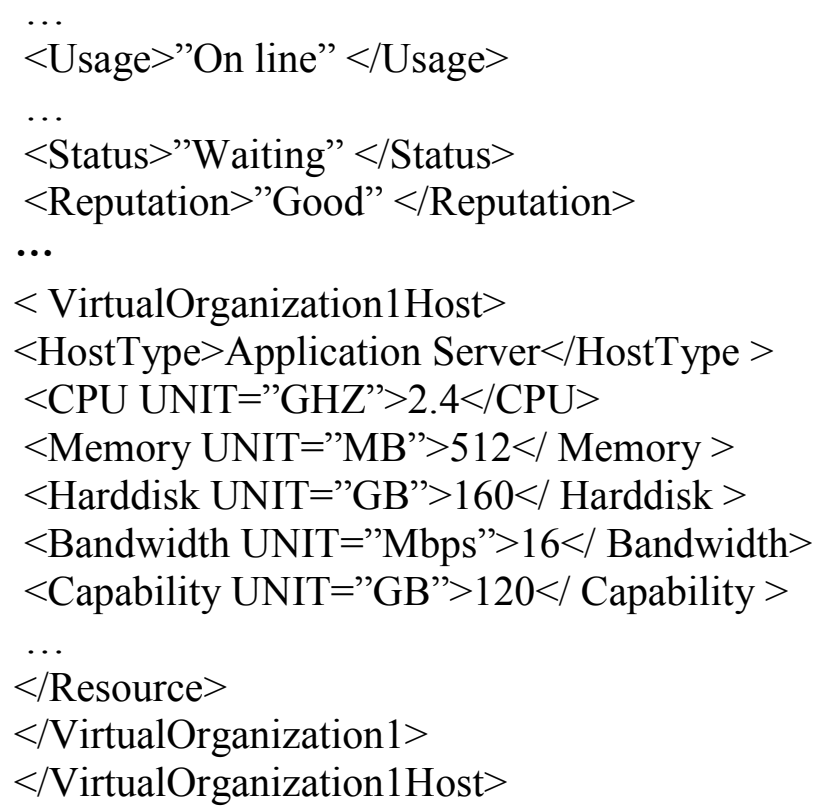

\section{Summary}

The work reported here on process preparation resource modeling is an extended part of DAPPS developed by the authors. This research seeks to establish systematic, collaborative and agile practice for process preparation in manufacturing grid. The next steps are to encapsulate the manufacturing resources into manufacturing grid as represented way in this paper. Findings from the ongoing investigation will be reported separately in the near future.

The main tasks of this research are as follows:

1. Process preparation resources are defined and classified.

2. Information modeling of process preparation in manufacturing grid is discussed.

3. The integrated service mode of process preparation resources in manufacturing grid is discussed.

4. An example of one kind of process preparation resource is given to illustrate the resource semantic description based on XML.

\section{Acknowledgements}

This work was financially supported by Postdoctoral Program of Science Foundation of Jiangsu Province of China (0901041C) and "excellence plans-zijin star" Foundation of Nanjing University of Science and Technology. The supports are gratefully acknowledged. The authors would also acknowledge the anonymous reviewers.

\section{References}

[1] Foster I, Kesselman C, Tuecke S, The anatomy of the grid. Int J Supercomput Appl. $15(2001) 1-21$.

[2] K. Keahey, M. Ripeanu, K. Doering, Dynamic Creation and Management of Runtime Environments in the Grid. Proceedings of Workshop on Designing and Building Web Services (GGF 9), Chicago, IL, October (2003) .

[3] Yifei Tong, Dongbo Li, A grid-based agile process preparation system. Int J Adv Manuf Technol. 35(2008)1219-1226.

[4] Ye Zuoliang, GU Xinjian, Qian Yadong, Manufacturing grid- the Applications of Grid Tech nology in Manufacturing Industry. CHINA MECHANICAL ENGINEERING.15 (2004) 1717 -1720.

[5] The Global Grid Forum Web Site,http://www.gridforum.org.

[6] E. Deelman, J. Blythe, Y. Gil, et al Mapping Abstract Complex Workflows onto Grid

Environments. Journal of Grid Computing.1(2003)25-39. 